\title{
Medicina transfusional en la pandemia de COVID-19. La visión del Centro Nacional de la Transfusión Sanguínea
}

\author{
Jorge Trejo-Gómora * ${ }^{*}$ José Salazar-Bailon ${ }^{1}$ \\ ${ }^{1}$ Centro Nacional de la Transfusión Sanguínea, Secretaría de Salud, Ciudad de México, México
}

\section{Resumen}

La pandemia originada por el coronavirus 2 del síndrome respiratorio agudo grave (SARS-CoV-2) ha sometido a una gran presión a los sistemas de salud de todo el mundo. Experiencias en brotes anteriores nos han enseñado que cuando los sistemas sanitarios se ven desbordados, la mortalidad por enfermedades para las que existen vacunas o tratamientos también puede aumentar drásticamente. Durante el brote de enfermedad por el virus del Ébola de 2014-2015, el exceso de fallecimientos por sarampión, paludismo, virus de inmunodeficiencia humana [VIH]/sida y tuberculosis atribuible al colapso de los sistemas sanitarios superó al de las muertes por ébola. Es por lo anterior que los sistemas de salud deben enfocar sus esfuerzos para mantener la continuidad de la atención de servicios médicos esenciales como son: el programa de vacunación, servicios de salud reproductiva (que incluyan la atención del embarazo, parto y el puerperio inmediato), salud en la infancia y en adultos mayores, enfermedades mentales, enfermedades crónicas no transmisibles (que incluyen cáncer y cardiovasculares), enfermedades infecciosas (VIH), tratamientos hospitalarios críticos, tratamiento de problemas urgentes de salud y servicios auxiliares como el diagnóstico básico por imagen, los servicios de laboratorio y los bancos de sangre. Entonces, un sistema de salud organizado y preparado es un sistema que cuente con la gerencia necesaria para mantener la continuidad de todas aquellas necesidades que requieran a la transfusión sanguínea como parte del tratamiento esencial (procesos oncológicos, atención de la hemorragia obstétrica, urgencias, traumatismos, anemias sintomáticas y cirugías de urgencia) y de aquellas otras situaciones en las cuales la transfusión de sangre o de algún componente se presente como una posible opción para el tratamiento de pacientes con la enfermedad por coronavirus 2019 (COVID-19) (plasma convaleciente en uso de investigación).

PALABRAS CLAVE: SARS-CoV-2. COVID-19. Plasma convaleciente. Transfusión sanguínea.

\section{Transfusion Medicine in the COVID-19 Pandemic. The vision of the National Center of Blood Transfusion}

\begin{abstract}
The pandemic caused by the severe acute respiratory syndrome coronavirus 2 (SARS-CoV-2) has put great pressure on health systems around the world. Experiences in previous outbreaks have taught us that when health systems are overwhelmed, mortality from diseases for which there are vaccines or treatments can also increase dramatically. During the 2014-2015 Ebola virus disease outbreak, the excess deaths from measles, malaria, HIV/AIDS, and tuberculosis attributable to the collapse of health systems exceeded that of Ebola deaths. For this reason, health systems should focus their efforts to maintain the continuity of care for essential medical services such as: the vaccination program, reproductive health services - which include care for pregnancy, childbirth and the immediate puerperium. - Health in childhood and the elderly, mental illnesses, chronic non-communicable diseases - including cancer and cardiovascular diseases - infectious diseases such as HIV, critical hospital

Correspondencia:

*Jorge Trejo-Gómora

E-mail: jorge.trejo@salud.gob.mx

Gac Med Mex. 2021;157(Supl3):S151-S157

Disponible en PubMed

www.gacetamedicademexico.com

0016-3813/@ 2021 Academia Nacional de Medicina de México, A.C. Publicado por Permanyer. Este es un artículo open access bajo la licencia CC BY-NC-ND (http://creativecommons.org/licenses/by-nc-nd/4.0/).
\end{abstract}


treatments, treatment of urgent health problems and auxiliary services such as basic imaging diagnosis, laboratory services and blood banks. An organized and prepared health system is a system that has the necessary management to maintain the continuity of all those needs that require blood transfusion as part of essential treatment (oncological processes, care of obstetric hemorrhage, emergencies, trauma, symptomatic anemias and emergency surgeries) and of those other situations in which the transfusion of blood or a component is presented as a possible option for the treatment of patients with coronavirus disease 2019 (COVID-19) (convalescent plasma in research use).

KEY WORDS: SARS-CoV-2. COVID-19. Convalescent plasma. Blood transfusion.

\section{Introducción}

En diciembre de 2019 se presentó en Wuhan (provincia de Hubei, de la República Popular China) un brote de neumonía de causa desconocida. Lo anterior derivó en una investigación por el país de tal forma que las autoridades de salud de la ciudad de Wuhan informaron a la Organización Mundial de la Salud (OMS) de la presencia de un conglomerado de 27 casos de síndrome respiratorio agudo de etiología desconocida'. Lo anterior llevó a que científicos chinos aislaran una nueva cepa viral que pertenece a los betacoronavirus del grupo $2 \mathrm{~B}$ con al menos un $70 \%$ de similitud en la secuencia genética con el coronavirus del síndrome respiratorio agudo grave (SARS-CoV), el cual se nombró por la OMS como 2019-nCoV. Siguiendo las mejores prácticas de la OMS para nombrar a las nuevas enfermedades infecciosas humanas, en colaboración y consulta con la Organización Mundial de Sanidad Animal y la Organización de las Naciones Unidas para la Agricultura y la Alimentación, la OMS ha denominado la enfermedad como COVID-19, abreviatura de enfermedad por coronavirus 2019 por sus siglas en inglés

\section{Preparación de los servicios de sangre en México, aspectos generales}

El riesgo de transmisión de la COVID-19 por transfusión de hemocomponentes y hemoderivados ahora es solo teórico y probablemente mínimo, pero experiencias anteriores con otros brotes virales sugieren que habrá un impacto negativo significativo en los suministros de sangre, debido a la donación reducida de sangre.

Es por lo anterior que los servicios de sangre deben tomar medidas para gestionar una respuesta de manera adecuada a la pandemia y considerar tempranamente el riesgo de desabasto de hemocomponentes, por lo que se deberá considerar los siguientes aspectos':
- Baja en disponibilidad de donantes de sangre.

- Mejorar el uso racional de la sangre.

- Evaluar el riesgo de escasez en suministro de materiales y equipos críticos.

- Gestión de recursos humanos en el servicio de sangre.

- Mitigación de exposición y contagio en los servicios de sangre.

- Seguridad y confianza de los donadores en transmisión local y comunitaria de enfermedades infecciosas.

\section{Transfusión sanguínea, uso racional de la sangre durante la pandemia de COVID-19}

De acuerdo con reportes de AABB (anteriormente conocida como American Association of Blood Banks) al 25 de febrero de 2020, no hay reportes asociados a transmisión de COVID-19 por medio de la transfusión.

Las células sanguíneas carecen de los receptores en tejido pulmonar y digestivo a los cuales se fija el virus, por lo que hoy en día la posibilidad es extremadamente baja ${ }^{2}$.

A pesar de ello, se ha definido que los métodos de inactivación de patógenos (tanto para plasma como concentrados plaquetarios) son eficientes para SARS$\mathrm{CoV}$, coronavirus causante del síndrome respiratorio de Oriente Medio (MERS-CoV) y coronavirus 2 del síndrome respiratorio agudo grave (SARS-CoV-2) ${ }^{3}$.

\section{Aspectos que considerar en inmunohematología}

Posterior a la revisión del metaanálisis de datos, no se reporta la presencia de anticuerpos en las pruebas de compatibilidad, sin embargo, existe la posibilidad teórica de producción de anticuerpos fríos por los mecanismos de infección asociados a inmunoglobulina $M$, por lo que se recomienda dar seguimiento de esta posibilidad en estos casos. 
En Italia se encontraron anticuerpos antieritrocitos en la mitad de los pacientes atendidos con COVID-19. Sin embargo, las características reportadas de la reactividad en la prueba directa de antiglobulina fue distinta a las observadas en anemias hemolíticas autoinmunes. La asociación con el virus no se ha elucidado completamente, pero es importante considerarlo, ya que podría deberse a anemias graves por la sepsis y a transfusiones reiteradas ${ }^{4}$.

\section{Recomendaciones de transfusión en paciente hospitalizados con infección por SARS-CoV-2}

En términos generales y en apego a los criterios restrictivos implementados mundialmente para los pacientes con anemia, podríamos considerar que la transfusión está considerada con los siguientes niveles de hemoglobina, como:

- Hemoglobina >10 g/dl. Inapropiada, salvo situaciones excepcionales (nivel de evidencia alto, recomendación fuerte). No existe evidencia sustancial de incremento en la supervivencia o algún otro efecto deseable aplicando criterios liberales de transfusión.

- Hemoglobina 8 a 10 g/dl. No indicada, pero podría ser considerada en algunos pacientes (anemia sintomática, hemorragia activa, pacientes hematooncológicos con sangrado y plaquetopenia).

- Hemoglobina 7 a $8 \mathrm{~g} / \mathrm{dl}$. Cuando hay signos 0 síntomas objetivos de incapacidad para satisfacer la demanda tisular de oxígeno, que podrían empeorar por la anemia (nivel de evidencia medio, recomendación fuerte).

- Hemoglobina 6 a 7 g/dl. La transfusión, en general, está indicada.

- Hemoglobina <6 g/dl. Transfusión recomendada salvo contraindicaciones excepcionales.

\section{Recomendaciones de transfusión de concentrado eritrocitario en el paciente en terapia intensiva y manejo ventilatorio por infección por SARS-CoV-2}

La recomendación es implementar, al igual que en el paciente hospitalizado, un criterio basado en inestabilidad hemodinámica incipiente y con nivel de hemoglobina $<7 \mathrm{~g} / \mathrm{dl}$.

Incluso con pacientes con inestabilidad asociada a sepsis, el criterio restrictivo se asocia a una exposición menor a transfusiones (hasta 3 unidades menos, sin diferencia en la mortalidad a 30 días).

\section{Recomendaciones de transfusión de concentrado eritrocitario en el paciente con insuficiencia respiratoria aguda por infección por SARS-CoV-2}

De acuerdo con los reportes internacionales, el descenso de hemoglobina en los pacientes COVID-19 que han ingresado a terapia intensiva con insuficiencia respiratoria aguda no ha sido clínicamente relevante ${ }^{5,6}$.

La mayoría de los pacientes se presentan con niveles normales de hemoglobina, y en pacientes en terapia intensiva, en promedio no mayor a $11 \mathrm{~g} / \mathrm{dl}$, con el nadir mínimo reportado en $10.2 \mathrm{~g} / \mathrm{dl}^{7}$.

- Se recomienda transfusión de concentrado eritrocitario (CE) en pacientes clínicamente enfermos con insuficiencia respiratoria que tienen concentración de $\mathrm{Hb}>5 \mathrm{~g} / \mathrm{dl}$ (recomendación fuerte, baja calidad de evidencia pediátrica).

- En pacientes críticamente enfermos con insuficiencia respiratoria que no tienen hipoxemia aguda grave, condición crónica que motive cianosis o anemia hemolítica y cuyo estado hemodinámico sea estable, se recomienda no administrar CE si la hemoglobina es $\geq 7 \mathrm{~g} / \mathrm{dl}$ (recomendación fuerte, evidencia de moderada calidad pediátrica).

- Si bien la evidencia clínica existente sobre la respuesta terapéutica es insuficiente para realizar una recomendación de transfusión en el contexto del paciente críticamente enfermo con insuficiencia respiratoria con concentraciones de hemoglobina entre menores de 7 y hasta $5 \mathrm{~g} / \mathrm{dl}$, es razonable la transfusión en este contexto basado en la valoración clínica de paciente $e^{7-11}$.

\section{Recomendaciones de transfusión en paciente con infección grave por SARS-CoV-2 y coagulación intravascular diseminada}

Si bien la hemorragia clínicamente significativa no es un evento común en el paciente COVID-19, cuando ocurre en el contexto asociado a coagulación intravascular diseminada (CID), el uso de productos sanguíneo debe ser considerado como en la coagulopatía por sepsis, con terapia objetivo como se menciona a continuación: 
- Transfundir plaquetas si están por debajo de 30,000 con el objetivo de tenerlas sobre las 50,000 en pacientes con sangrado activo. Considerar detener la anticoagulación si el paciente requiere productos sanguíneos, valorando riesgo contra beneficio.

- Si hay sangrado activo, proveer productos sanguíneos. Plasma fresco congelado entre 15 y $20 \mathrm{ml} / \mathrm{kg}$ en pacientes con sangrado activo 0 con tiempo de protrombina y tiempo de tromboplastina parcial activado prolongados ( $>1.5$ veces lo normal). Si hay disponibilidad de ellos, emplear concentrados del complejo de protrombina.

- En pacientes con hipofibrinogenemia < $1.5 \mathrm{~g} / \mathrm{l}$, administrar crioprecipitados o concentrado de fibrinógeno (si está disponible) $)^{12}$.

\section{Recomendaciones de transfusión en paciente con infección grave por SARS-CoV-2 que requiere procedimiento de oxigenación por membrana extracorpórea}

Según el consenso de expertos en la materia, los pacientes que requieren oxigenación por membrana extracorpórea (ECMO) pueden ser atendidos con sistema venovenoso, con disminución de los niveles límite $(7 \mathrm{~g} / \mathrm{dl})$, ya que pueden ser bien tolerados si se encuentran estables y sin sangrado activo.

Dicho procedimiento debe ser proporcionado por equipos experimentados en apego a los lineamientos de la OMS.

Los niveles límite más altos (8 a $10 \mathrm{~g} / \mathrm{dl})$ podrían ser considerados en pacientes en estado de choque o evidencia de entrega de oxígeno alterada a pesar del manejo con ECMO.

No deben realizarse sistemáticamente esfuerzos para mantener niveles fisiológicos de hemoglobina.

Por otra parte, los protocolos de transfusión con niveles más bajos, asociados a técnicas de preservación de sangre ( $p$. ej., autotransfusión del circuito durante la descanulación) podrían disminuir las necesidades transfusionales por paciente ${ }^{13}$.

\section{Consideraciones adicionales}

El empleo de monitorización fisiológica continua en el paciente gravemente enfermo proporciona valores hemodinámicos que permiten tomar decisiones clínicas, entre los cuales están los signos vitales, la oximetría de pulso y el cálculo de índices de aporte de oxígeno o el consumo de oxígeno tisular. En general se considera que los valores fisiológicos son apropiados para la toma de decisiones en transfusión.

Sin embargo, el empleo de biomarcadores (como el nivel de lactato o el pH intragástrico) no han mostrado evidencia de ser predictores de requerimiento transfusional, dosis requerida 0 de respuesta al tratamiento ${ }^{14}$.

\section{Recomendaciones de transfusión en paciente con infección grave por SARS- CoV-2 y cáncer hematológico}

Si bien el consenso generalizado es mantener criterios restrictivos, este abordaje podría no ser el apropiado para poblaciones con alto riesgo de desarrollar complicaciones por anemia, sobre todo el paciente con asociación de neoplasia mieloide, el adulto mayor y la cardiopatía.

Esto ha planteado controversias incluso dentro de una misma institución, sin embargo, dada la situación actual de disminución de la donación a lo largo de la pandemia, se deberá adoptar dichos criterios restrictivos bajo las siguientes premisas:

- Incrementar el intervalo entre transfusiones y optimizar la dosis de CE, sí es posible, para disminuir el número de visitas hospitalarias.

- En el caso de las plaquetas, disminuir su uso profiláctico en el caso de procedimientos menores o cirugías programadas, las cuales podrían incluso diferirse si no son urgentes

- En el caso del paciente ambulatorio, emplear cifras de 10,000 plaquetas por microlitro para la profilaxis en pacientes con requerimientos crónicos.

- Así mismo, considerar el empleo de antifibrinolíticos de forma temprana en pacientes sin COVID-19 con riesgo de sangrado ${ }^{15}$.

\section{Implicaciones de la medicina transfusional como coadyuvante en el tratamiento de la COVID-19}

\section{Enfermedad trombótica}

Si bien la incidencia no está bien definida, la tromboembolia venosa está debida a mecanismos multifactoriales: respuesta inflamatoria sistémica, estasis, postración del paciente, daño endotelial directo del virus, fijación a receptores de la enzima convertidora de angiotensina 2 (ACE2). 
Reportes de autopsia en pacientes en Wuhan han mostrado microtrombos en la vasculatura pulmonar, lo cual puede contribuir al desequilibrio ventilación/ perfusión o a cambios hidrostáticos que generan edema.

Los datos obtenidos en los reportes en Wuhan permiten sugerir que la heparina de bajo peso molecular o la heparina no fraccionada pueden ser benéficas en aquellos pacientes con COVID-19 y niveles de dímero D seis veces por encima del límite normal alto.

Por tanto, un gran porcentaje de los pacientes que ingresen a unidad de cuidados intensivos requerirá anticoagulación profiláctica, salvo que haya una contraindicación tácita para ello ${ }^{8-11}$.

\section{Coagulación intravascular diseminada}

Al inicio de la crisis sanitaria, un estudio reporta 16 de 183 pacientes hospitalizados en Wuhan, sin embargo, los estudios más recientes reportan una asociación clara entre infección por SARS-CoV-2 y coagulopatía; y la CID es común en el paciente que fallece por esta causa.

Por ello, se puede asociar directamente la causa, y la gran mayoría de estos pacientes cumple con las definiciones del tercer Consenso de Sepsis. Además, la estancia a largo plazo en cama y el empleo de esteroides se asocia a tromboembolia venosa en casos graves.

La media de tiempo en que se presenta es a los cuatro días del ingreso hospitalario.

Se requiere evaluar cambios en los parámetros de coagulación, productos de la degradación de fibrina y dímero $D$ con disfunción multiorgánica, con evidencia de microangiopatía trombótica.

Se deben considerar siempre los criterios CID de la Sociedad Internacional de Trombosis y Hemostasia.

En el reporte más reciente de la Sociedad Internacional de Trombosis y Hemostasia se encontró que el uso de heparina (especialmente de bajo peso molecular) en pacientes con criterios CID $\geq 4$ o con dímero $D$ seis veces superior a lo normal se asocia a mejor pronóstico ${ }^{5,6}$.

\section{TRATAMIENTO}

Como en cualquier caso de CID, la evaluación es multisistémica y continua, con evaluación de la presencia y o gravedad de la trombosis o del sangrado; necesidad de realizar procedimientos invasivos 0 cirugía y resultados del laboratorio (con mediciones repetidas 2-3 veces al día).

El segundo paso tradicionalmente se ha considerado tratar la causa de base que genera el desequilibrio hemostático. En el contexto que nos ocupa, mientras se dispone de evidencia sobre un tratamiento específico, podrá ser necesario soportar las alteraciones de la hemostasia hasta limitar el sangrado activo.

Pacientes sin trombosis ni sangrado. No está indicada la corrección de valores anormales, excepto en casos con deterioro extremo que preanuncie sangrado inminente.

Respecto a los antifibrinolíticos, no se administran sistemáticamente. En casos con hiperfibrinólisis demostrada o en sangrado refractario a tratamiento, considerar ácido tranexámico. Suele bastar con una dosis única. En sangrado menor o local, considerar antifibrinolíticos orales o tópicos.

Considerar la profilaxis con heparina/heparina de bajo peso molecular, en especial cuando se trate de pacientes críticamente enfermos, pacientes con sepsis o puérperas.

Suspender en caso de sangrado o laboratorio de riesgo (plaquetas menores de 15,000 o fibrinógeno $<100$ ). Valorar de acuerdo con la función renal al momento de valoración el cambio de heparina de bajo peso molecular por heparina no fraccionada.

En pacientes con sepsis disponemos de evidencia indirecta, aunque creciente, de que la heparina tendría efectos beneficiosos más allá del efecto anticoagulante: modula la inflamación y protege al glucocálix en endotelio.

El soporte transfusional, en general, no difiere de las recomendaciones generales de productos mencionadas anteriormente y siempre dependerá de la evaluación clínica dinámica, constante y orientada a la presentación clínica que resolver y las metas que lograr ${ }^{12}$.

\section{Síndrome de tormenta de citocinas}

La evidencia cumulativa sugiere que un subgrupo de pacientes desarrolla un síndrome de tormenta de citocinas. El síndrome hemofagocítico secundario es una entidad subdiagnosticada, que consiste en un síndrome hiperinflamatorio caracterizado por hipercitocinemia fulminante y fatal con falla multiorgánica. En el caso de su asociación a COVID-19 es mediante el incremento de la interleucina 2, interleucina 7, factor estimulante de colonias de granulocitos, interferón 
gamma, proteína 10 inducible y factor de necrosis tumoral alfa, entre otras.

Estudios retrospectivos muestran que los niveles altos de ferritina y de interleucina 6 se encontraron consistentemente como predictores de fatalidad, lo cual sugiere asociación directa con hemofagocitosis.

Por ello, se sugiere considerar dicha sospecha diagnóstica, y para pacientes críticamente enfermos con alta respuesta inflamatoria, el recambio plasmático terapéutico puede eliminar una gran cantidad de mediadores inflamatorios y mejorar el estado de alta respuesta inflamatoria del paciente. Con el fin de mejorar la condición de falla orgánica múltiple en pacientes críticos, es un tratamiento adyuvante eficaz para pacientes críticos.

Se conocen los efectos del recambio plasmático terapéutico para eliminar sustancias patogénicas en el plasma de los pacientes. En la pandemia por SARS y MERS se ha empleado para depurar citocinas y frenar la tormenta de citocinas asociada, con lo cual se reduce el daño tisular y se reduce el daño por radicales libres.

Si bien la indicación por COVID-19 no está incluida en las guías de la ASFA (American Society for Apheresis), las consecuencias fisiopatológicas bien podrían ser atendidas con este procedimiento de forma oportuna en los servicios clínicos ${ }^{16}$.

\section{Plasma convaleciente}

Más allá de la atención de apoyo, actualmente no hay opciones de tratamiento comprobadas para la COVID-19, la neumonía relacionada, la infección causada por el SARS-CoV-2 y la respuesta inflamatoria desencadenada.

El plasma convaleciente humano representa ahora una opción para el tratamiento de la COVID-19 basada exclusivamente en protocolos de investigación que pretenden conocer la eficacia y seguridad de la transfusión de plasma convaleciente con títulos de anticuerpos neutralizantes anti-SARS-CoV-2 en pacientes con COVID-19 grave.

En el caso del SARS-CoV-2, el mecanismo de acción anticipado por el cual la terapia pasiva de anticuerpos mediaría la protección es la neutralización viral, sin embargo, otros mecanismos pueden ser posibles: citotoxicidad celular dependiente de anticuerpos y fagocitosis.

El pasado 20 de abril del 2020 el Centro Nacional de la Transfusión Sanguínea en coordinación con la Comisión Federal Para la Protección Contra Riesgos
Sanitarios emitieron el lineamiento técnico de uso de plasma convaleciente para tratamiento de COVID-19 grave en ensayos clínicos, basado en la experiencia internacional, la contingencia nacional desencadenada por la expansión del SARS-CoV-2 y en una extensa búsqueda y análisis bibliográfico que ya emergía derivado de la experiencia internacional y nacional sobre la COVID-19.

\section{Terapia con células mesenquimales}

Al día de hoy, la erradicación sistémica del virus depende del propio sistema inmunitario del paciente. Cuando el sistema inmunitario hiperactivado elimina al virus, produce una cantidad muy alta de factores inmunitarios, lo cual conduce a la tormenta de citocinas.

Recientes investigaciones muestran que las células mesenquimales son negativas a los receptores de ACE2 y serina proteasa transmembrana 2, y secretan factores antiinflamatorios que previenen la tormenta de citocinas.

Por tanto, las células mesenquimales poseen propiedades de inmunomodulación con las cuales se puede prevenir la producción masiva y no controlada de citocinas proinflamatorias, inhibir las respuestas inmunitarias y reducir el daño inmunológico a tejidos y órganos. Incluso pueden reemplazar y reparar el tejido que ha sufrido daño e inhibir la fibrosis pulmonar. Se ha empleado en paciente con COVID-19 con resultados que se han considerado buenos.

El mecanismo involucrado es la disminución en la diferenciación de células dendríticas convencionales a células $\mathrm{CD}_{3}{ }^{+}$, induciendo la diferenciación de células dendríticas plasmacitoides por medio de la prostaglandina E2 e inducción de producción de células dendríticas regulatorias dependientes de interleucina 10.

Por tanto, la terapia con células progenitoras hematopoyéticas podría suprimir la activación excesiva del sistema inmunitario, promover la reparación endógena al mejorar el microambiente, detener la progresión de la inflamación pulmonar y aliviar los síntomas de la insuficiencia respiratoria. Los estudios iniciales muestran que es una terapia segura y efectiva en este contexto clínico ${ }^{17}$.

\section{Conflicto de intereses}

Los autores declaran no tener conflicto de interés alguno con el tema de la publicación. 


\section{Financiamiento}

No se recibió financiamiento ni patrocinio por ninguna organización o institución.

\section{Responsabilidades éticas}

Protección de personas y animales. Los autores declaran que para esta investigación no se han realizado experimentos en seres humanos ni en animales.

Confidencialidad de los datos. Los autores declaran que han seguido los protocolos de su centro de trabajo sobre la publicación de datos de pacientes.

Derecho a la privacidad y consentimiento informado. Los autores declaran que en este artículo no aparecen datos de pacientes.

\section{Bibliografía}

1. Listas de verificación sobre planificación para bancos de sangre y servicios de transfusión en caso de transmisión comunitaria de COVID-19 [Internet]. México: Centro Nacional de la Transfusión Sanguínea; marzo de 2020. Disponible en: https://www.gob.mx/cms/uploads/attachment/ file/542999/Listas de verificaci n BS y ST CORONAVIRUS_13032020-C.pdf

2. Chang L, Zhao L, Gong H, Wang Lunan, Wang L. Severe acute respiratory syndrome coronavirus 2 RNA detected in blood donations. Emerg Infect Dis. 2020:26(7):1631-3.

3. Chang L, Yan Y, Wang L. Coronavirus disease 2019: Coronaviruses and blood safety. Transfus Med Rev. 2020;34(2):75-80.

4. Berzuini A, Bianco C, Paccapelo C, Bertolini F, Gregato G, Cattaneo A et al. Red cell bound antibodies and transfusion requirements in hospitalized patients with COVID-19. Blood. 2020;136(6):766-8.
5. Fan BE, Chong VCL, Chan SSW, Lim GH, Lim KGE, Tan GB, et al. Hematologic parameters in patients with COVID-19 infection. Am J Hematol. 2020 Jun;95(6):E131-E134.

6. Ruan Q, Yang K, Wang W, Jiang L, Song J. Clinical predictors of mortality due to COVID-19 based on an analysis of data of 150 patients from Wuhan, China. Intensive Care Med. 2020;46(5):846-8.

7. Huang C, Wang Y, Li X, Ren L, Zhao J, Hu Y, Clinical features of patients infected with 2019 novel coronavirus in Wuhan, China. Lancet. 2020;395:497-506.

8. Valentine SL, Bembea MM, Muszynski JA, Cholette JM, Doctor A, Spinella PC, et al. Consensus Recommendations for RBC transfusion practice in critically ill children from the Pediatric Critical Care Transfusion and Anemia Expertise Initiative. Pediatr Crit Care Med. 2018;19:884-98.

9. Mueller MM, Van Remoortel H, Meybohm P, Aranko K, Aubron C, Burger $\mathrm{R}$, et al. Patient blood management recommendations from the 2018 Frankfurt Consensus Conference. JAMA. 2019;321(10):983-97.

10. Carson JL, Guyatt G, Heddle NM, Grossman BJ, Cohn CS, Fung MK, et al. Red blood cell transfusion thresholds and storage. JAMA 2016;316(19):2025-35

11. Doctor A, Cholette JM, Remy KE, Argent A, Carson JL, Valentine SL, et al. Recommendations on RBC transfusion in general critically ill children based on hemoglobin and/or physiologic thresholds from the $\mathrm{Pe}$ diatric Critical Care Transfusion and Anemia Expertise Initiative. Pediatr Crit Care Med. 2018:19(9S Suppl 1):S98-S113.

12. Bikdeli B, Madhavan MV, Jimenez D, Chuich T, Dreyfus I, Driggin E, et al. COVID-19 and thrombotic or thromboembolic disease: Implications for prevention, antithrombotic therapy, and follow-up: JACC State-of-theArt Review. J Am Coll Cardiol. 2020;75(23):2950-73.

13. Ramanathan K, Antognini D, Combes A, Paden M, Zakhary B, Ogino M, et al. Planning and provision of ECMO services for severe ARDS during the COVID-19 pandemic and other outbreaks of emerging infectious diseases. Lancet Respir Med. 2020;8(5):518-26.

14. Liu XM, Wang DQ. [Consideration and suggestions on development of blood transfusion department under the epidemic situation of novel coronavirus pneumonia]. Zhonghua Yi Xue Za Zhi. 2020;100(14):1041-3.

15. Zeidan AM, Boddu PC, Patnaik MM, Bewersdorf JP, Stahl M, Rampal RK, et al. Special considerations in the management of adult patients with acute leukaemias and myeloid neoplasms in the COVID-19 era: recommendations from a panel of international experts. Lancet Hematol. 2020;7(8):e601-e612.

16. Li Y, Liu S, Zhang S, Ju Q, Zhang S, Yang Y, et al., Current treatment approaches for COVID-19 and the clinical value of transfusion-related technologies. Transfus Apher Sci. 2020;59(5):102839.

17. Leng Z, Zhu R, Hou W, Feng $Y$, Yang $Y$, Han Q, et al. Transplantation of ACE2 mesenchymal stem cells improves the outcome of patients with COVID-19 pneumonia. Aging Dis. 2020;11(2):216-28. 